\title{
Photoluminescence of ortho-bromobenzophenone
}

\author{
A.A. Avdeenko, O.S. Pyshkin, V.V. Eremenko, M.A. Strzhemechny, \\ L.M. Buravtseva, and R.V. Romashkin \\ B. Verkin Institute for Low Temperature Physics and Engineering \\ of the National Academy of Sciences of Ukraine, 47 Lenin Ave., Kharkov 61103, Ukraine \\ E-mail: strzhemechny@ilt.kharkov.ua
}

Received August 3, 2006

\begin{abstract}
Phosphorescence spectra of crystalline ortho-bromobenzophenone (2-bromobenzophenone, $2 \mathrm{BrBP}$ ) were measured from $1.6 \mathrm{~K}$ to room temperature. A cardinal emission mechanism crossover occurs within this temperature range. At low temperature the phosphorescence spectrum is a superposition of two similar sets of equidistant bands spaced by the $\mathrm{C}=\mathrm{O}$ stretch frequency. We ascribe these two sets to the emission of two different conformers, one of which is metastable and do not manifest itself above approximately $70 \mathrm{~K}$. Presence of two conformers is explained by the fact that the $2 \mathrm{BrBP}$ molecule deforms considerably upon excitation. The emission from the stable conformer survives up to approximately $140 \mathrm{~K}$ or even higher. At roughly $60 \mathrm{~K}$ and higher, another type of emission reveals itself in the shape of a two very broad partly overlapping bands, which gains in intensity with increasing temperature. Above $150 \mathrm{~K}$ only this two-hump feature is observed in phosphorescence spectra. Additional phosphorescence experiments were carried out to elucidate the nature of this spectrum, including phosphorescence measurements of $2 \mathrm{BrBP}$ in ethanol solutions as well as time-resolved and varying-excitation-intensity measurements from crystals. Based on results of all the experiments reported here and on our own single-crystal x-ray structure data we conclude that the above two-band spectrum recorded near room temperature is due to the emission of single-photon bimolecular triplet excimer formed by the carbonyl groups of two neighbor 2-bromobenzophenone molecules. It is got the first time that a bimolecular excimer has been observed and reliably identified in a benzophenone derivative solid.
\end{abstract}

PACS: 78.55.Kz, 31.50.+w

Keywords: photoluminescence, benzophenone, excimer, triplet exiton.

\section{Introduction}

It is more than a century that crystals of benzophenone and its derivatives attract attention and are utilized as a model system for studying photophysics and photochemistry of $n \pi^{*}$ and $\pi \pi^{*}$ excited triplet states [1], polymorphism and overcooled liquid [2,3], vitreous states [4], energy transport by triplet excitons [5]. Benzophenone and its derivatives are widely used in pharmacy (c.f., for instance, [6]), nonlinear optics [7-10], and in light-emitting diodes [11,12]. The absolute quantum yield of benzophenone phosphorescence is the phosphorescence standard [13].

The unsubstituted benzophenone molecule, which is the most studied one among carbonyl compounds, change inconsiderably upon excitation [14,15]. It was more so interesting to study the photophysical pro- perties of the 2-bromobenzophenone (2BrBP) molecule, because theoretical calculations [16] show that the $2 \mathrm{BrBP}$ molecule changes its conformation upon photoexcitation drastically. In addition, as the structure studies [17] imply, the molecular packing in the solid has an important peculiarity, namely, closely spaced (about $3.4 \AA$ ) parallel $\mathrm{C}=\mathrm{O}$ group pairs, which underexcitation are prone to form emitting autocompexes of various types (for example, excimers [18]).

Physically, the benzophenones represent the group of so-called flexible molecules. Their butterfly shape is a result of the balance between the conjugation energy and the repulsive interaction of the contact between the species at phenyl-ring positions $2 a$ and $2 b$ as shown in the molecular scheme in Fig. 1. The conjugation 


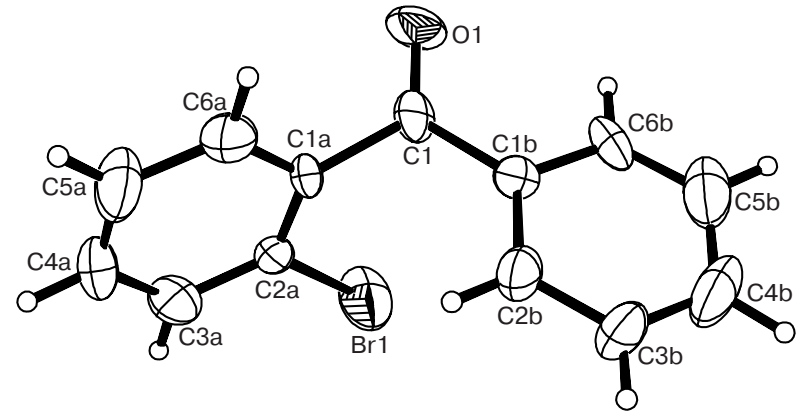

Fig. 1. The ortho-bromobenzophenone molecule in the crystal environment [17] at room temperature with 50\%-probability displacement ellipsoids. The highly asymmetric shape of the molecule can be imagined with the unsubstituted ring very roughly in the same plane with the $\mathrm{CO}$ group and the substituted ring roughly normal to that plane.

energy depends strongly on the distribution of electron charges over the molecule. When packed in a crystal array, all the benzophenone substitution derivatives suffer conformational changes due to crystal-field interactions and packing-related steric restrictions. These changes are the more pronounced, the more conformationally compliable is the molecule, which depends crucially on the substitution species and their positions on the phenyl rings. In this study we chose 2-bromobenzophenone (2BrBP) for the following reasons. First, the $2 \mathrm{BrBP}$ molecule with the substitution bromine at the repulsion-contact position is more conformationally mobile [17] compared to other benzophenones previously studied. Second, we expected solid 2BrBP to behave in an unusual way, because its molecular shape both in the gas and in the solid is highly asymmetric in contrast to any other bromo-substituted compounds studied previously [19]. Third, because of its abnormal crystallization behavior and by analogy with pristine benzophenone, which is also reluctant to crystallize [20] we suspected that $2 \mathrm{BrBP}$ can easily form glass.

The ultimate goal of any physical study is to establish microscopic mechanisms behind any macroscopic response of a solid. In this study we carried out a combined investigation of photoluminescence and structural properties of $2 \mathrm{BrBP}$, supported by relevant quantum-chemical calculations, to understand the basic causes behind the luminescence spectra as a function of temperature. Temperature variations were not only a general background but were also utilized as an instrument to bring to forefront this or that emission mechanism. We understood that the microscopic relation between structure and intrinsic emission of 2BrBP molecules in crystal environment as the main aim of study could be reached only in ultimately pure samples, which were prepared in as accurate way as possible to reduce not only the concentration of impurities but also that of crystal defects such as dislocations, grain boundaries, etc. To this end, we first purified the source material as shown below in the corresponding section.

The structure of the paper is as follows. Experimental details are in the next section. After that we summarize our recently published structure results [17] with a stress on the peculiarities needed to better understand the relation between photoluminescence and structure. Phosphorescence results are presented in the next section, together with a discussion that involves our structure data [17] and quantum-chemical calculations [16]. The last section sums up the main conclusions. Part of the results related to the high-temperature phosphorescence spectra, which are due to excimer emission has been published in a brief communication [21].

\section{Experimental}

\subsection{Sample preparation}

The material of 2-bromobenzophenone from two commercial sources (Yugsintez Company in Dnepropetrovsk, Ukraine, and Factory of Chemical Reactants in Shostka, Ukraine) were preliminarily purified by multiple recrystallization from ethanol solution. Final purification was performed by absorption chromatography, the immovable phase being aluminum oxide. Crystals of ortho-bromobenzophenone shaped as transparent colorless plates were grown from ethanol solutions. The typical dimensions of the crystals selected for luminescence studies were $1 \times 5 \times 8 \mathrm{~mm}$. Larger crystals of size circa $10 \times 10 \times 15 \mathrm{~mm}$ were grown from a seed immersed into supercooled melt $[10,22,23]$. The seed was cut from a crystal prepared with the former technique. Luminescence spectra were identical from crystals grown using both techniques.

\subsection{Luminescence measurements}

For phosphorescence spectra measurements we employed an automated apparatus which incorporates a DFS-12 double monochromator with an inverse dispersion of $5.2 \AA / \mathrm{mm}$. The emission from the crystal was recorded with a cooled FEU-106 photomultiplier in the photon counting regime. Phosphorescence spectra were corrected for the varying spectral sensitivity of the recording equipment. The spectral slit width during measurements did not exceed $0.5 \AA$. Sample excitation was performed with the aid of a LGI-21 pulsed nitrogen laser with a generation wavelength of $337.1 \mathrm{~nm}$, pulse duration $10 \mathrm{~ns}$, pulse recurrence frequency $100 \mathrm{~Hz}$, and power flux 
$20 \mathrm{~kW} / \mathrm{cm}^{2}$. The emission power of the laser can be varied using calibration filters.

Phosphorescence spectra were recorded within a wide temperature range from 1.6 to $293 \mathrm{~K}$. The temperature was maintained and stabilized to within $\pm 1 \mathrm{~K}$ with the aid of a helium flow cryostat. The temperature of the sample was varied using an electric heater; temperature stabilization was effected by a special electronic system; the sample temperature was measured by a semiconductor resistance pickup.

\section{Structure}

The molecular and crystal structure determined for the stable monoclinic polymorph by single-crystal x-ray diffraction have been published elsewhere [17]. In this Section we provide information necessary for the interpretation of optical results.

The shape of the $2 \mathrm{BrBP}$ molecule in the crystal (cf. Fig. 1), as found from single-crystal x-ray structure determination [17], is characterized by a strong asymmetry of the torsion angles of the two phenyl rings. At room temperature, the two dihedral torsion angles are $\mathrm{O} 1-\mathrm{C} 1-\mathrm{C} 1 \mathrm{a}-\mathrm{C} 6 \mathrm{a}=68.3(5)^{\circ}$ and $\mathrm{O} 1-\mathrm{C} 1-\mathrm{C} 1 \mathrm{~b}-\mathrm{C} 6 \mathrm{~b}=-17.6(6)^{\circ}$. Compared to other single or double halogen-para-substituted benzophenones and their polymorphs, for example [24-28], the torsion angle difference is very large. Similar torsion angle asymmetry was found in ortho-chlorobenzophenone [29].

As quantum-mechanical calculations for the isolated molecule in the ground state show [17], the torsion angle asymmetry is an intrinsic property of the molecule. However, comparison of these calculations (see also Table 1 below) with the experimental findings for the molecular structure in the solid shows that both torsion angles change upon crystallization, substantially more than in the single or double substituted para-benzophenones. It means that in the case of $2 \mathrm{BrBP}$ we deal with a compound which is conformationally labile. Indeed, as one can infer from Fig. 2, the energy barrier between the two conformers ( $\mathrm{Br}$ at positions 2 and 6), if compared to room temperature or unsubstituted benzophenone [14], is unusually low. Irrespective of the minor differences in the results of two calculation methods, the main conclusion is that in the room-temperature gas the occupancy of the $6 \mathrm{Br}$ isomer state should be high. This conclusion is also true for solutions, though perhaps to a lesser extent. In the solid the steric restrictions forbid the molecule in the ground state to flip easily to the $6 \mathrm{Br}$ state. Nevertheless, the latent isomeric instability is pronounced, which manifests itself in many ways. Thus, the melting point of $2 \mathrm{BrBP}\left(42{ }^{\circ} \mathrm{C}\right)$ [30] is appreciably lower than that of the 4-bromo-

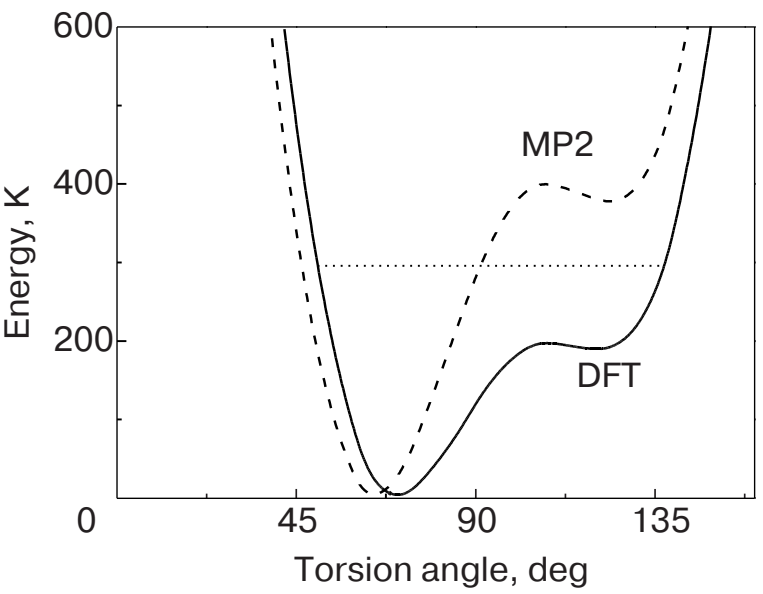

Fig. 2. The total energy of an isolated 2-bromobenzophenone molecule versus the torsion angle of the substituted ring, $\omega_{a}$; all other parameters have been optimized for every ${ }^{0} a$ value. This plot is a blowup of the previously published figure [17]. The dotted horizontal line symbolizes room temperature.

benzophenone isomorph $\left(82.5^{\circ} \mathrm{C}\right)$ [30]. Second, the melt exhibits a stubborn reluctance to crystallize and, hence, can be supercooled easily. Presumably, the same properties render the sublimation crystallization technique impracticable: the deposit at the cool end of the column refuses to solidify and stays liquid (at least, to the bare eye). Third, even in the crystal state 10-15 degrees below the melting point, samples are prone to becoming spontaneously partly liquid or highly amorphous (possibly, due to spurious temperature gradients). Fourth, as will be shown below, photoluminescence exhibits a wealth of unusual properties, which essentially differ from those in (quasi)symmetric benzophenones.

We should also mark the following feature of the crystal structure of $2 \mathrm{BrBP}$ (see Fig. 3), which is important for understanding the spatial distribution of emitting molecular fragments. There are two dif-

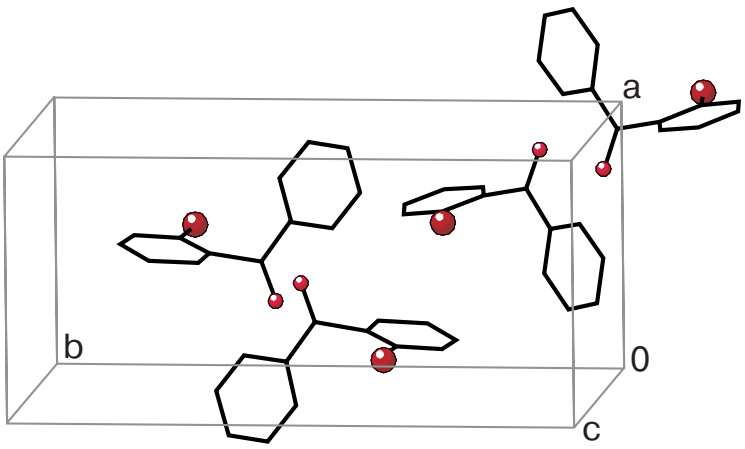

Fig. 3. The elementary cell with four molecules, showing orientations of the two «paired» parallel CO groups. Every pair is linked by an inversion operation. Between themselves the pairs are linked by a rotation operation. 
ferently oriented systems of paired CO groups, which are undoubtedly responsible for at least part of the phosphorescence emission. The distance between the two parallel CO bonds in a pair is quite short (3.4 $\AA$ ). The angle between the two different groups is $23.80^{\circ}$.

\section{Results and discussion}

Even the first phosphorescence spectra measured on 2-bromobenzophenone at room temperature (see Fig. 4) were strikingly different from similar spectra from the 4-substituted analog [31], which suggested cardinally different mechanisms that control light emission in these seemingly similar crystals. Hereinafter, unless stated otherwise, all spectra are normalized so that the maximum intensity recorded is set to unity.

In order to investigate into this difference more closely, we measured phosphorescence spectra at ten temperatures from 1.6 to $293 \mathrm{~K}$, as presented in Fig. 5. Inspection of the ten traces shows that the spectra suffer cardinal transformation, suggesting a crossover between emission mechanisms and/or emitting centers. Even at the lowest temperature of $1.6 \mathrm{~K}$ the spectrum contains two sets, one of the two barely discernable. Each set consists of at least four (or five) virtually equidistant (in energy) lines. The weak set lights up with increasing temperature. The spectral distance ( $\left.\simeq 1680 \mathrm{~cm}^{-1}\right)$ between the separate bands in both sets is the known $\mathrm{C}=\mathrm{O}$ stretch-mode frequency. These spectra has two properties, which are uncommon for benzophenone derivatives in the solid. First, all bands in both sets are unusually broad at any temperature: in unsubstituted benzophenone and $4 \mathrm{BrBP}$ the respective bands at low temperatures are appreciably narrower and richly structured.

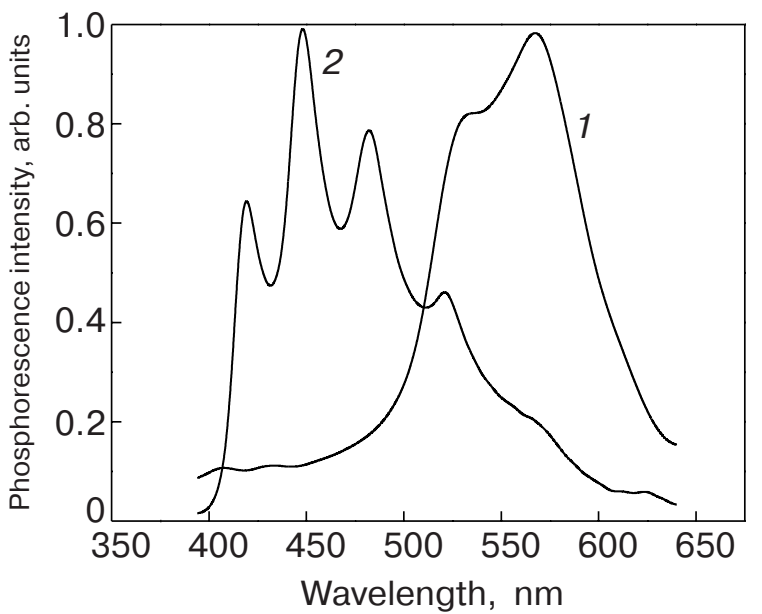

Fig. 4. Comparison of the phosphorescence spectra at room temperature from $2 \mathrm{BrBP}$ (1) and $4 \mathrm{BrBP}$ (2) [31]. Notice the absence of the $\mathrm{C}=\mathrm{O}$ stretch repetition in the 2BrBP spectrum.

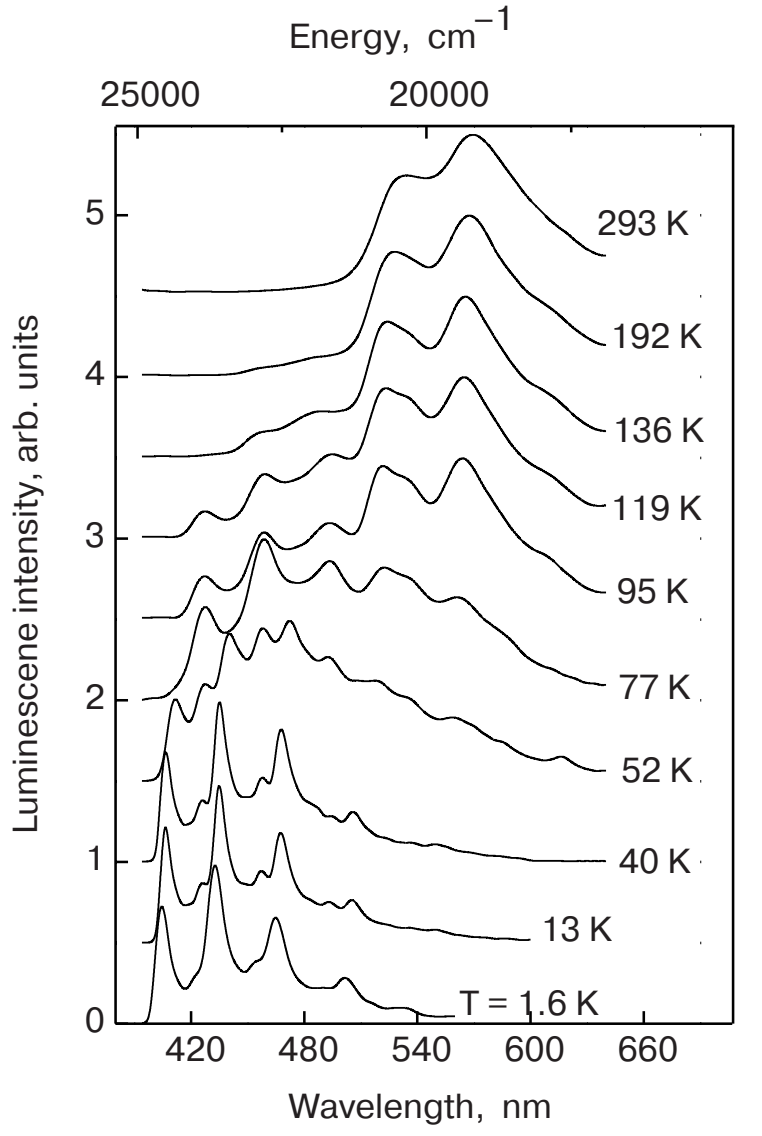

Fig. 5. Phosphorescence spectra of monoclinic ortho-bromobenzophenone measured at ten temperatures as indicated in the plot. The spectra are shifted upward to avoid overcrowding.

As the temperature increases from 1.6 to $52 \mathrm{~K}$, the dimmer low-energy set of equidistant bands increases in intensity. Both sets widen with increasing temperature but, as the temperature is raised from 52 to $77 \mathrm{~K}$, the high-energy set disappears while the other set grows further in intensity and goes on broadening. The energy positions of the bands in both sets have a tendency to shift to the red with increasing temperature. As follows from Fig. 5, reminiscences of progressively broadening bands of the high-energy set can be discerned in the spectra up to $180 \mathrm{~K}$ and above.

At as low as $77 \mathrm{~K}$, a new broad feature starts to form, growing in intensity with temperature. One can easily identify this feature as a doublet, because the shoulder at the red side of the high-energy feature looks like one of the dying components of the lowenergy equidistant set. At room temperature, only two broad bands survive.

Making use of the available structure data, $a b$ initio calculations, as well as additional optical results, we will try to explain the anomalies observed in phosphorescence spectra of 2-bromobenzophenone. 


\subsection{Character of triplet exciton state}

The general form of the low-temperature phosphorescence spectrum of $2 \mathrm{BrBP}$ crystals, namely, the equidistant character and the vibron repetition energy of about $1680 \mathrm{~cm}^{-1}$ allows us to conclude that the emitting center is the ketone group. To determine the character of the emitting center, it is a common practice to use the known rule [32], which prescribes a relation between the triplet character and the twist angles. This rule, which is meaningful for benzophenone derivatives, both twist angles of which are (almost) equal. This rule is completely inapplicable for $2 \mathrm{BrBP}$, because the two twist angles quoted above indicate «opposite» $\left(n \pi^{*}\right.$ versus $\left.\pi \pi^{*}\right)$ characters. Therefore, we rely our conclusion on a different consideration. The character of the $T_{1}$ triplet state in $4 \mathrm{BrBP}$ [33] is $n \pi^{*}$. On the other hand, the lowtemperature phosphorescence spectrum of $4 \mathrm{BrBP}$ [31] is qualitatively similar to that of $2 \mathrm{BrBP}$ (the lowermost curve in Fig. 5). Thus, we conclude that the character of the emitting triplet exciton center in solid 2-bromobenzophenone is $n \pi^{*}$, at least at low temperatures.

The conclusion of the preceding paragraph is, however, in shear contradiction with the absorption spectrum of $2 \mathrm{BrBP}$ at low temperatures as shown in Fig. 6. Usually, the positions of the $S_{0}-T_{1}$ band in absorption and phosphorescence spectra coincide for a few wavenumbers, at most. This is so in pristine benzophenone [34], 44'-dichlorobenzophenone [35], and 4-bromobenzophenone [31], but not in solid 2-bromobenzophenone. As one can see in Fig. 6, the absorption spectrum of $2 \mathrm{BrBP}$ does not contain a distinct band responsible for the transition to the $T_{1}$ triplet state with $n \pi^{*}$ character and only a singletsinglet absorption is present. As to a similar transition to $\pi \pi^{*} T_{1}$, even if present, it would not have revealed itself because the respective oscillator strength is a few orders of magnitude lower as compared to that of the transition to a $n \pi^{*}$ triplet [36]. Hence we come to the inference that the lower triplet state in an unexcited molecule, which is ready to accept a photon, is of $\pi \pi^{*}$ character, which is at variance with the above reliable conclusion that emission occurs from a triplet with $n \pi{ }^{*}$ character.

Certain understanding of the nature of the above paradox comes if we consider the respective molecular conformations, as calculated [16] using the (RO)B3LYP / 6-31G package for its (singlet) ground and (triplet) excited states of $2 \mathrm{BrBP}$ as opposed to

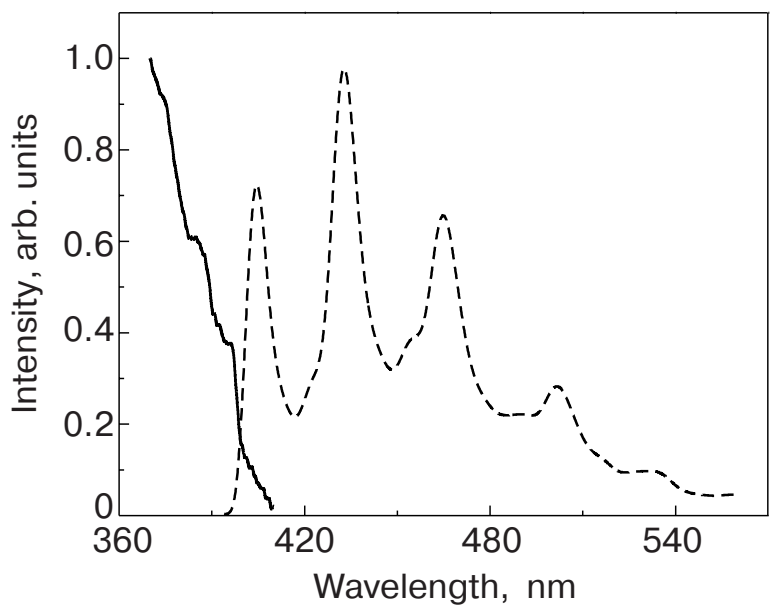

Fig. 6. The 2BrBP absorption spectrum (solid trace) in comparison with the phosphorescence spectrum (broken trace) at the same temperature of $4.2 \mathrm{~K}$.

similar quantities in the para-substituted analog (see Table 1). The main conclusions that follow from this Table are as follows. The torsion angles of the substituted and unsubstituted rings in the para-isomer do not change essentially when the molecule goes from the ground to excited state. But in the ortho-isomer this change is catastrophic, namely, the torsion angle difference (which is large in the ground state), roughly speaking, changes sign retaining its magnitude. In other words, upon excitation the molecule wants to flip over to a cardinally different conformer state. From Table 1 follows that the lowest triplet in this conformer must have character $n \pi^{*}$. Indeed, this can be inferred from both a longer $\mathrm{C}=\mathrm{O}$ bond and a substantially decreased dipole moment. This trend coincides with that for the para-isomer in Table 1 and it is reliably established [33] that the emitting triplet in $4 \mathrm{BrBP}$ is of character $n \pi^{*}$.

Table 1. Ab initio calculated properties of the ground and triplet excited states for isolated molecules of para and ortho Br-substituted benzophenones*.

\begin{tabular}{l|c|c|c|c}
\hline \hline \multirow{2}{*}{\multicolumn{1}{c|}{ Property }} & \multicolumn{2}{|c|}{ Para-isomer } & \multicolumn{2}{c}{ Ortho-isomer } \\
\cline { 2 - 5 } & singlet & triplet & singlet & triplet \\
\hline \hline C=O bond length $(\AA)$ & 1.226 & 1.328 & 1.222 & 1.325 \\
Dipole moment (Dy) & 2.794 & 2.135 & 2.966 & 1.950 \\
Torsion angles (deg) & & & & \\
substituted ring & 25.0 & 21.1 & 57.7 & 0 \\
nonsubstituted ring & -26.3 & -24.4 & -10.3 & -42 \\
\hline \hline
\end{tabular}

* Owing to intrinsic computation difficulties, the characteristics of the excited state are calculated to a poorer accuracy. 


\subsection{Low-temperature phosphorescence}

The molecular shape of the $2 \mathrm{BrBP}$ molecule in its unexcited and excited states as well the molecule energetics depicted in Fig. 2 allow us to explain the two anomalies observed in the phosphorescence spectra at temperatures ranging from 1.6 to $100 \mathrm{~K}$, namely, presence of two equidistant sets of CO-stretch repetitions as well as the comparatively large width of separate bands.

As discussed above, the equilibrium shape of the excited molecule differs drastically from that in the ground state. Therefore, upon excitation and subsequent internal conversion the molecule abruptly finds itself in a state, which corresponds only to a local energy minimum, while the global minimum is separated by a barrier, which is determined by the crystal environment restrictions; we expect the height of that barrier to be close to that depicted in Fig. 2. If the temperature is low enough, it is this metastable state that will be a predominant emitter. Only a minor fraction of excited molecules manage to «break through» to the global minimum state, which will emit with a considerable red shift. Thus, two sets of $\mathrm{C}=\mathrm{O}$-stretch-repeated bands will appear in the phosphorescence spectrum. As the temperature is increased, more and more molecules reach the global minimum. As a result, the intensity of the fingerprint set from the lowermost $n \pi{ }^{*}$ state increases at the expense of the emission from the metastable term.

As was noted above, the shape and energetics of the 2BrBP molecule (cf. Fig. 2) suggest a strongly enhanced conformational pliability of the molecule compared to other benzophenone derivatives mentioned above. In other words, the $2 \mathrm{BrBP}$ molecule is soft with respect to torsional oscillations of both phenyl rings. Therefore, given the absence of absorption data, we can only surmise that it is these low-frequency oscillations that could be the reason behind the abnormally large bandwidths even at low temperatures.

\subsection{Phosphorescence at higher temperatures}

The two-hump feature that appears above $80 \mathrm{~K}$ (Fig. 5) should be attributed to excimer emission [21]. A few arguments confirm this conclusion. First, the shift with respect to the lowermost band of the fingerprint set by $5500-6000 \mathrm{~cm}^{-1}$, which is reasonable for the excimer binding energy. Second, in order to elucidate this problem, we measured phosphorescence from $2 \mathrm{BrBP}$ in ethanol solution. In Fig. 7 we plot spectra for two $2 \mathrm{BrBP}$ concentrations. At low concentrations, the spectrum is an almost unstructured broad band positioned roughly within the range where the intrinsic emission at low temperatures is concentrated. At elevated 2BrBP concentrations,

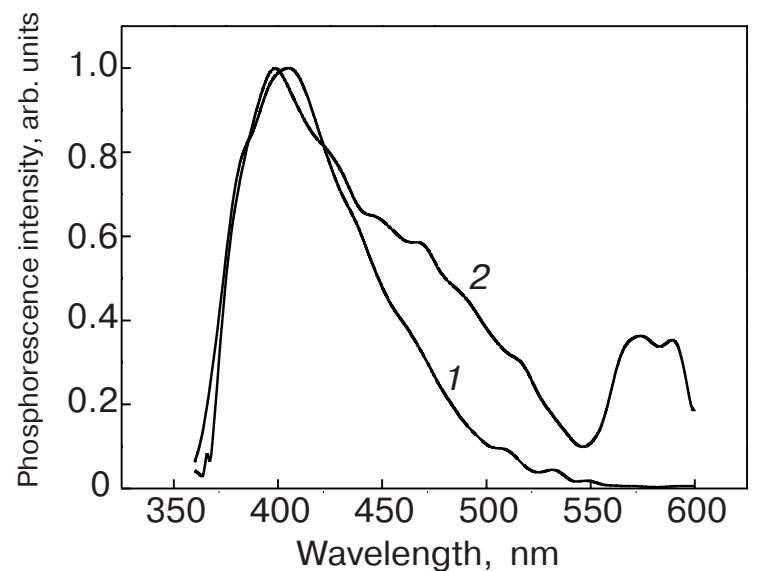

Fig. 7. Phosphorescence spectra from 2-bromobenzophenone in ethanol solutions at room temperature; $10^{-4} \mathrm{~mol} / \mathrm{l}(1)$, $10^{-2} \mathrm{~mol} / \mathrm{l}(2)$. A new feature appears at higher concentrations.

when the number of $2 \mathrm{BrBP}$ dimers in the solution increases considerably, the spectrum develops a twohump feature. The shape and position (between 550 and $600 \mathrm{~nm}$ ) of this feature virtually coincide with those of the feature in the phosphorescence spectrum of solid 2BrBP at room temperature (Fig. 5). Third, since in all cases when an excimer is the predominant emitter, the phosphorescence is, as a rule, a relatively long process.

In order to understand better the nature of the room-temperature double-hump feature, we measured time-resolved spectra with a time window of $\tau=$ $=50 \mu \mathrm{s}$. The spectrum measured immediately after the excitation pulse (shown in Fig. 8 as a solid curve) is shifted to shorter wavelengths compared to all delayed spectra, one of which is plotted as a dashed curve. The no-delay spectrum is clearly structured. The nature of this structure is not clear but it is the intrinsic emission that is presumable responsible for this short-time part of the spectrum. The subsequent spectra (like the broken curve in Fig. 8), taken after multiples of $\tau$, are weaker and decay but slowly with increasing delay time. Note that the seemingly more intensive no-delay spectrum contribute very little compared to the double-hump spectrum, which persists up to a tenth of second. Such a long-lived emission can be possible in two cases. In one case, presence of rather deep traps is needed, which a triplet exciton reaches at varying distances from the excitation center. Then, inevitably, it is implicitly assumed that exciton transport is efficient. However, it is unlikely in view of the fact that the shape of an excited molecule differs dramatically from that before excitation. As a result, a very large «polaron» effect must render the coherent exciton band width too narrow to ensure sufficiently fast exciton transport. In 


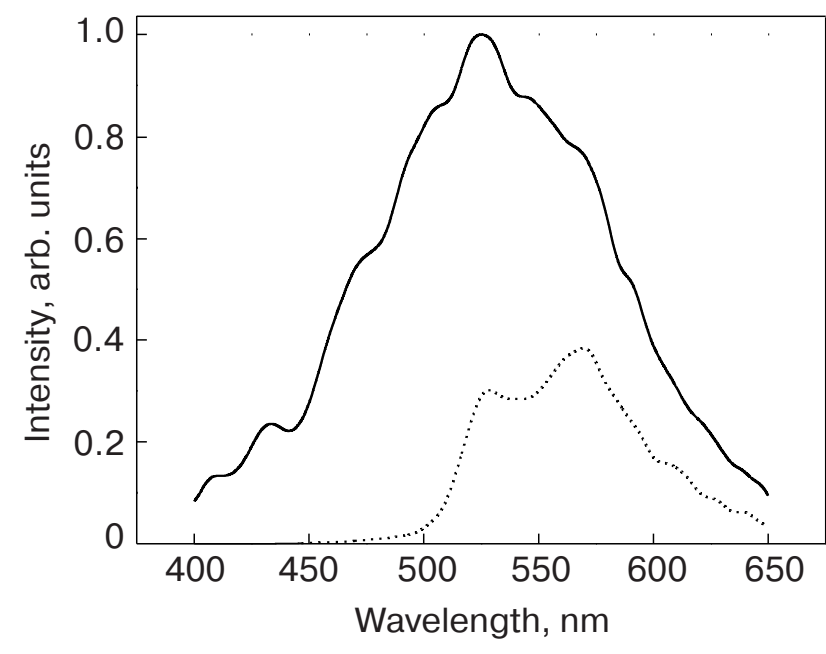

Fig. 8. Time-resolved room-temperature phosphorescence spectra from the ortho-bromobenzophenone crystal. Solid curve: the recording strobe duration $50 \mu \mathrm{s}$; no delay. Dashed curve: the strobe duration $50 \mu \mathrm{s}$; the delay time $300 \mu$ s. To make relative intensity clearer, both spectra are normalized to the maximum intensity of no-delay spectrum.

the other case, which we believe applies to the situation under discussion, the emission is due to an excimer with a sufficiently large binding energy. We also remind that the crystal structure of $2 \mathrm{BrBP}$ has a specific peculiarity (see Fig. 3), namely, the $\mathrm{C}=\mathrm{O}$ groups form closely spaced $(3.4 \AA)$ pairs. Such a configuration is favorable for the formation of an bimolecular excimer.

To further elucidate the nature of the high-temperature phosphorescence, we studied how the emission intensity of the two-hump feature (the uppermost trace in Fig. 5) varies with pumping intensity. We made these measurements separately for the two com-

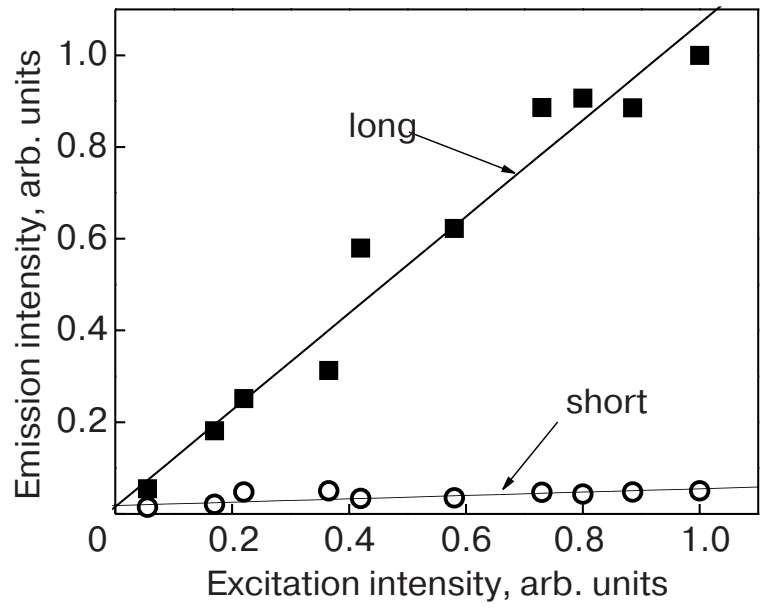

Fig. 9. The emission intensities of the short-wave and long-wave excimer emission components versus excitation intensity. ponents that constitute this two-hump feature. In Fig. 9 we plot the emission intensities in both components versus the excitation intensity. One can see that the long-wave and short-wave components behave differently. However, the emission intensities of both are linear functions of the excitation intensity. This is evidence that a single excitation is needed to promote the formation of the excimer emission [18].

\section{Conclusions}

1. Phosphorescence spectra of crystalline orthobromobenzophenone have been measured over the temperature range from 1.6 to $300 \mathrm{~K}$ and the spectral range from 15000 to $26000 \mathrm{~cm}^{-1}$.

2. The phosphorescence spectra at low temperature ( 1.6 to ca $60 \mathrm{~K}$ ) exhibit two sets of equidistant bands (spaced by about $1680 \mathrm{~cm}^{-1}$ ) shifted one with respect to another by approximately $1100 \mathrm{~cm}^{-1}$. Each of these two sets is a fingerprint signature, typical of lowtemperature phosphorescence of any benzophenone derivative. The spectrum of this form is evidence that the character of the emitting excited triplet state is $n \pi^{*}$.

3. Absorption spectra over the range, where the equidistant phosphorescence set is revealed, show no bands responsible for transitions from the ground state to a triplet of character $n \pi^{*}$. Given this finding as well as the fact that transitions to $\pi \pi^{*}$ states are strongly forbidden we make the inference that the lowermost triplet state in the unexcited molecule of $2 \mathrm{BrBP}$ is of character $\pi \pi^{*}$.

4. The above paradox is resolved if we take into consideration the results of quantum-chemistry calculations of the molecule in the triplet excited state. Upon excitation the molecule changes its shape dramatically flipping a dramatically different conformation, which by all criteria (the changes in $\mathrm{C}=\mathrm{O}$ bond length and dipole moment) is of character $n \pi^{*}$.

5 . The above-mentioned excitation-promoted change of the molecular shape explains the presence of two similar equidistant sets in the low-temperature phosphorescence spectra. Indeed, right after excitation the molecule finds itself in a conformation, which in the new situation is metastable. However, the global minimum is separated by an energy barrier, which should be overcome through thermal activation. As the temperature is increased, such emissionless transitions become more frequent, which is corroborated by the behavior of the low-temperature spectra with varying temperature.

6. The two-band feature, which appears at about $100 \mathrm{~K}$ and persists up to the melting point, is emission from a single-photon bimolecular excimer. This conclusion rests on a few facts and considerations, among 
which the most convincing one is provided by the phosphorescence measurements from 2BrBP solutions in ethanol: at comparatively high $2 \mathrm{BrBP}$ concentrations there appears a two-hump feature resembling the one in phosphorescence spectra from the crystal. The excimer nature of the room-temperature spectrum is confirmed by other pieces of evidence, which include time-resolved phosphorescence data and general considerations stemming from the structural and spectroscopic findings. Summing up, it is for the first time that a single-photon bimolecular excimer has been observed and reliably interpreted for a benzophenone derivative in the solid and in solutions.

The authors are sincerely grateful to A.V. Luzanov for his calculations of the excited triplet state of the ortho-bromobenzophenone molecule. The authors also thank V.N. Baumer for helpful discussions and P.V. Zinoviev for constructive criticism of the manuscript.

1. N.J. Turro, Modern Molecular Photochemistry, University Science Books, Sausalito (1991).

2. H. Cang, V.N. Novikov, and M.D. Fayer, J. Chem. Phys. 118, 2800 (2003).

3. D.J. Graham, P. Magdalinos, and M. Tosi, J. Phys. Chem. 99, 4757 (1995).

4. N.A. Davydova, V.I. Mel'nik, J. Baran, and M. Drozd, J. Molec. Structure 651-653, 171 (2003).

5. R.M. Hochstrasser, J. Chem. Phys. 40, 1038 (1964).

6. H. Hsieh, J. Liou, Y. Lin, N. Mahindroo, J. Chang, Y. Yang, Sh. Chen, U. Tan, Ch. Chang, T. Chen, Ch. Lin, Y. Chang, and Ch. Wang, Bio-Organic and Medic. Chem. Lett. 13, 101 (2003).

7. D. Lammers, K. Betzler, D. Xue, and J. Zhao, Phys. Status Solidi A180, R5 (2000).

8. B. Zhao, W. Lu, Z. Zhoua, and Y. Wu, J. Mater. Chem. 10, 1513 (2000).

9. J. Hulliger, A.A. Kaminskii, and H.J. Eichler, Adv. Funct. Mater. 11, 243 (2001).

10. W. Wang, W. Huang, Ya. Ma, and J. Zhao, J. Crystal Growth 270, 469 (2004).

11. S. Hoshino and H. Suzuki, Appl. Phys. Lett. 69, 224 (1999).

12. S. Hoshino and H. Suzuki, Pure Appl. Chem. 71, 2095 (1999).

13. Handbook of Organic Photochemistry, J.C. Scaiano (ed.), CRC Press, Boca Raton (1989), vol. 1, p. 237.

14. R. Hoffmann and J.R. Swenson, J. Phys. Chem. 74, 415 (1970).

15. G. Hilburn, R.K. Power, K.A. Marti, and A.M. Nishimura, Chem. Phys. Lett. 100, 429 (1983).

16. Part of those calculations have been presented as an oral talk elsewhere: A.A. Avdeenko, L.M. Buravtseva,
O.A. Zhykol, A.V. Luzanov. O.S. Pyshkin, and M.A. Strzhemechny, Abstracts of International Conference on Specrtoscopy of Molecular Crystals, Chernogolovka, Russia (2004), p. 15.

17. V.N. Baumer, R.V. Romashkin, M.A. Strzhemechny, A.A. Avdeenko, O.S. Pyshkin, R.I. Zubatyuk, and L.M. Buravtseva, Acta Crystallogr. E61, o1170 (2005).

18. N.J. Turro, M. Aikawa, and I.R. Gould, J. Am. Chem. Soc. 104, 856 (1982).

19. P. Dais, Z. Phys. Chemie 168, 43 (1990).

20. H. Cang, V.N. Novikov, and M.D. Fayer, J. Chem. Phys. 118, 2800 (2003).

21. M.A. Strzhemechny, A.A. Avdeenko, V.V. Eremenko, O.S. Pyshkin, and L.M. Buravtseva, accepted to Phys. Chem. Lett.

22. D.E. Ovsienko, G.A. Alfintsev, G.P. Chemerinsky, S. Budurov, and N. Stoichev, J. Crystal Growth 60, 107 (1982)

23. H. Kutzke, H. Klapper, R.B. Hammond, and K.J. Roberts, Acta Crystallogr. B56, 486 (2000).

24. F.J. Zúñiga and A. Criado, Acta Crystallogr. B51, 880 (1995).

25. V.V. Mitkevich, V.G. Lirtsman, M.A. Strzhemechny, A.A. Avdeenko, and V.V. Eremenko, Acta Crystallogr. B55, 799 (1999).

26. S. Ebbinghaus, D. Abeln, and M. Epple, Z. Kristallogr. 212, 339 (1997).

27. M.A. Strzhemechny, V.N. Baumer, A.A. Avdeenko, O.S. Pyshkin, R.V. Romashkin, and L.M. Buravtseva, submitted to Acta crystallogr. B.

28. B. Perić and B. Kojić-Prodić, Acta Crystallogr. C36, $211(2000)$.

29. A.G. Pinkus, K.K. Klausmeyer, R.P. Feazell, L.Y.C. Meng, and T.C. Chang, J. Chem. Crystallogr. 34, 637 (2004).

30. Handbook of Chemistry and Physics, 66-th Edition, CRC Press, Boca Raton (1986).

31. O.S. Pyshkin, A.A. Avdeenko, L.M. Buravtseva, and M.A. Strzhemechny (in preparation).

32. M. Lipson, P.F. McGarry, I.G. Koptyug, H.A. Staab, N.J. Turro, and D.C. Doetschman, J. Phys. Chem. 98, 7504 (1994).

33. W.J. Leigh, E.C. Lathioor, and M.J. StPierre, J. Am. Chem. Soc. 118, 12339 (1996).

34. V.I. Melnik and M.T. Shpak, Optika Spectrosc. (USSR) 29, 610 (1970).

35. A.A. Avdeenko, T.L. Dobrovolskaya, V.A. Kulchitskii, and Yu.V. Naboikin, Phys. Status Solidi B90, 405 (1980).

36. J.A. Barltrop and J.D. Coyle, Excited States in Orga nic Chemistry, John Wiley and Sons, London (1976). 